\title{
Abandono de atendimento psicológico por crianças em uma clínica-escola'
}

\author{
Psychology treatment dropout of children in a school-clinic
}

Tatiane Regina dos Santos Cunhaa ${ }^{[a]}$, Silvia Pereira da Cruz Benetti ${ }^{[b]}$

\footnotetext{
${ }^{[a]}$ Mestre em Psicologia Clínica pela Universidade do Vale do Rio dos Sinos, (Unisinos), São Leopoldo, RS - Brasil, e-mail: taticunha@pop.com.br

${ }^{[b]}$ Doutora em Child and Family Studies pela Syracuse University (SYR), Estados Unidos, professora na Universidade do Vale do Rio dos Sinos, (Unisinos), São Leopoldo, RS Brasil, e-mail:

sbenetti@unisinos.br
}

Recebido: $13 / 07 / 2011$

Received: $07 / 13 / 2011$

Aprovado: 16/11/2011 Approved: 11/16/2011

\section{Resumo}

O objetivo deste estudo é examinar, entre crianças que estiveram em atendimento psicológico numa clínica-escola, os fatores associados ao abandono psicoterápico. Para tal, foram analisados protocolos de atendimentos de crianças de 3 a 12 anos de idade, no período de 1999 a 2006, no arquivo de uma clínica-escola localizada na região metropolitana de Porto Alegre (RS). Ao todo, 499 protocolos foram analisados e observou-se que entre as crianças atendidas 136 abandonaram o tratamento. Os achados apontam que as crianças que mais abandonam são encaminhadas, em sua maioria, por escolas, possuem entre 6 a 9 anos de idade, de ambos os sexos, morando com a mãe somente. Os motivos de procura mais comuns entre essas crianças foram: problemas emocionais, de comportamento e dificuldades no ambiente escolar. Concluímos que se fazem necessários novos formatos de intervenções clínicas, voltados para qualidade da aliança terapêutica com os pais, principalmente nos momentos iniciais do processo terapêutico.

Palavras-chave: Psicoterapia. Crianças. Abandono.

\begin{abstract}
The objective of this study is to examine, among children referred for psychological assistance in a university clinic in the Metropolitan Region of Porto Alegre, Rio Grande do Sul State, Brazil,, the factors associated to psychotherapy dropout. The files of children between 3 to 12 years old seen at the clinic between the years of 1999 to 2006 were analyzed. A total of 499 protocols were investigated and it was identified that 136 dropped out of treatment. The results point out that children referred by the school, were 6 to 9 years old, both males and females and living with their mothers only were the groups that most frequently dropped out. The reasons for seeking psychotherapy in the drop out group were emotional and behavioral problems, and difficulties in the school environment. It was concluded that new forms of clinic interventions geared to the quality of the therapeutic alliance with parents.
\end{abstract}

Keywords: Psychotherapy. Child. Interruption.

1 Este artigo faz parte da dissertação de mestrado em Psicologia Clínica no Programa de Pós-Graduação do curso de Psicologia da Universidade do Vale do Rio dos Sinos (Unisinos) pela mestre Tatiane Regina dos Santos Cunha. Financiamento: Comissão de Aperfeiçoamento de Pessoal de Nível Superior Capes/Prosup 


\section{Introdução}

Investigar a clínica psicológica em relação aos locais de atendimento, à eficácia e aos resultados obtidos no trabalho terapêutico contribui para o desenvolvimento e qualidade das psicoterapias. Dessa forma, estudos voltados para o aprimoramento do trabalho clínico são de suma importância para a evolução, qualidade do atendimento e satisfação no âmbito dos atendimentos psicológicos oferecidos, respondendo aos objetivos fundamentais de promover a saúde biopsicossocial do indivíduo (Russel \& Orlinsky, 1996).

Um aspecto pouco investigado em relação à prática clínica é o abandono de terapia. Alguns autores (Chilelli \& Enéas, 2000; Löhr \& Silvares, 2006) sugerem que as pesquisas sobre a eficácia terapêutica não deveriam se restringir aos casos que finalizam os atendimentos, sendo importantes, igualmente, as investigações que focalizam as interrupções precoces do processo psicoterápico. Assim como os atendimentos finalizados por alta, os abandonos de tratamentos podem fornecer medidas para a efetividade dos atendimentos psicológicos e informações importantes sobre o processo psicoterápico. Consequentemente, investigar os abandonos de terapia, além de contribuir para uma maior compreensão dos fatores envolvidos no trabalho clínico, oferece subsídios para o aprimoramento técnico dos terapeutas e, principalmente, no caso específico, dos terapeutas em formação (Lhullier, 2002).

É expressivo o índice de desistência ou abandono psicoterápico em clínicas-escola (Lohr \& Silvares, 2006) e são escassos os estudos relativos ao tema (Chilelli \& Enéas, 2000). Dessa maneira, a investigação sobre a questão do abandono de psicoterapia se reveste de maior complexidade, quando associada aos locais que formam profissionais. 0 ensino e o treinamento de alunos, bem como a responsabilidade de oferecer um atendimento eficaz e ético, são aspectos inerentes a estes locais. Portanto, os abandonos psicoterápicos em instituições de formação servem como um alerta que pode indicar caminhos para a eficácia das psicoterapias, do ensino, da pesquisa, da formação e do tratamento em si (Lhullier, 2002).

Alguns trabalhos sobre abandono de tratamento psicoterápico por adultos identificam diversas razões para as interrupções associadas às questões financeiras, ao abuso de substâncias químicas, a características interpessoais e institucionais, entre outros aspectos (Melo \& Guimarães, 2005). Porém, Vargas (2002) ressalta que a maioria dos pacientes abandona o tratamento sem apresentar razões ao terapeuta. Em relação a crianças e a adolescentes, são poucos os estudos sobre essas populações, ainda que essas faixas etárias constituam a maior demanda por atendimento e apresentem, também, o maior índice de abandonos em psicoterapia (Godman, 2003; Prebianchi \& Cury, 2005; Kelleher, Ward-Estes \& Hayes, 2006; Louzada, 2003). Lhullier (2002), em um estudo sobre abandono de psicoterapia numa clínica-escola, encontrou entre as crianças da amostra um índice de 51,8\% referente aos abandonos de tratamento, dados similares aos de Luk, Staiger, Mathai, Wong, Birleson e Adler (2001), Louzada (2003) e Silvares (2006a). Segundo a literatura, pacientes que dependem da iniciativa de terceiros, tais como as crianças, apresentam menor aderência ao tratamento (Bueno, Córbora, Escolar, Carmona \& Rodrigues, 2001; Kazdin, 1990). Dessa forma, observa-se que alguns aspectos constituem peculiaridades relacionadas às características do atendimento psicoterápico infantil.

Dentre as inúmeras especificidades relacionadas aos atendimentos psicológicos para crianças, temos: o nível de dependência da criança, o tipo e intensidade das expectativas que os pais têm em relação ao filho, o quanto os pais se vêem envolvidos no problema do filho, as condições dos pais em tolerar mudanças, as possibilidades de estabelecimento da aliança terapêutica, as condições psíquicas dos envolvidos ao processo e o nível de desenvolvimento da criança. Nesse sentido, essas situações podem se constituir como dificuldades no estabelecimento da aliança terapêutica nessas faixas etárias, em razão da diversidade de fatores envolvidos no processo psicoterápico (Green, 2005).

Em termos de intervenção, nos atendimentos psicoterápicos para crianças e adolescentes, está presente a necessidade de participação direta de familiares e de outros sistemas, como professores ou outros profissionais. Green (2005) relata que resultados pobres em psicoterapia com crianças podem estar associados a pais com dificuldades de organização ou com desordens emocionais, além de outros fatores psicossociais. Os pais buscam o tratamento para seus filhos e são os formuladores da queixa que efetiva o pedido de tratamento para a criança, bem como são aqueles que cessam o tratamento, quando acharem que devem, sendo este um 
dos motivos de abandono. Quando ocorre a remissão dos sintomas na criança, fator que incentiva a busca de tratamento psicológico ao filho, existe uma tendência de os pais interromperem o tratamento por terem alcançado seu objetivo, a "melhora do sintoma" (Lhullier, 2002).

Dessa forma, os temas deste estudo, "abandonos de atendimentos psicológicos por crianças" e "clínicas-escola", focalizam aspectos relevantes do trabalho clínico relativos ao desenvolvimento de estudos sobre psicoterapia e o aprimoramento do trabalho nas clínicas-escola. Nesse ultimo caso, fundamenta-se no duplo papel que este espaço de formação de alunos deve cumprir em relação à relevância para a comunidade e à formação de profissionais. Assim, considera-se importante investigar as razões que levam as crianças a abandonarem seus atendimentos psicológicos, considerando tanto as especificidades do atendimento com crianças como as variáveis institucionais relacionadas aos abandonos.

\section{Metodologia}

\section{Delineamento}

A opção metodológica para o desenvolvimento da pesquisa foi quantitativa, descritiva e retrospectiva, caracterizando-se por um levantamento em arquivos. Dessa maneira, investigaram-se os prontuários do arquivo permanente de todas as crianças inscritas, no período de 1999 a 2006, em uma clínica-escola localizada na região metropolitana de Porto Alegre. A clínica foi fundada no ano de 1999, localizando-se em uma cidade com aproximadamente 270 mil habitantes, carente de serviços em saúde mental para a população em geral. Esse local atende, em média, 112 pacientes por ano e oferece quatro vagas para estágio por semestre para alunos curriculares em Psicologia Clínica, com opções de abordagens teóricas diferenciadas, incluindo Psicoterapia de Orientação Analítica, Psicoterapia Cognitivo-comportamental e Psicoterapia Fenomenológica Existencial. 0 serviço não é gratuito; cobra-se uma taxa única, de baixo valor, para todos os atendidos. Em caso de necessidade, os pacientes podem ser isentados do pagamento. 0 atendimento segue uma ordem de lista de espera, ordem de renda familiar e casos mais emergentes podem ser priorizados.
Participantes

Durante o período de 1999 a 2006, 899 pacientes, de diferentes faixas etárias, inscreveram-se na clínica-escola. Um total de 499 casos envolvia a população infantil. Dessas 499 crianças, 287 não receberam atendimento e 212 foram atendidas. A amostra foi composta por todos os protocolos de crianças de 3 a 12 anos de idade, de ambos os sexos, que realizaram atendimento psicológico individual e abandonaram o atendimento. Considerando esses critérios, a amostra final foi constituída por 136 (64,2\%) crianças que abandonaram o atendimento psicológico. Foi considerado abandono de psicoterapia quando a criança ou seu responsável, tendo comparecido a pelo menos uma sessão psicoterápica na clínica-escola, cessou o atendimento, independente do motivo que a levou a fazê-lo (Lhullier, Nunes \& Lessa, 2006; Piper, Ogrodniczuk, Joyce, McCallum, Rosie, O'Kelly et al., 1999). E, considerou-se alta, quando o terapeuta e o paciente - ou responsáveis concordaram e combinaram o encerramento do tratamento, independente do número de sessões utilizadas para isso.

\section{Procedimentos de coleta e análise dos dados}

Após a autorização para a realização da pesquisa, foram feitos encontros para discussão e planejamento do trabalho de coleta nos prontuários mantidos pela clínica, referentes a todos os atendimentos realizados. Os pacientes atendidos passam primeiro por uma triagem e, após a avaliação inicial, são encaminhados para psicoterapia. Os dados são registrados em prontuários específicos, contendo informações de caráter sociodemográfico e da evolução do caso, incluindo desde informações sobre o encaminhamento, a avaliação psicológica e o tratamento. No presente estudo foram obtidos dados dos pacientes em relação a: aspectos sociodemográficos (sexo, idade, escolaridade); dados da família (idade dos pais, escolaridade dos pais, renda familiar, estrutura familiar); aspectos de procura de atendimento (motivo de procura, locais de encaminhamento, número de pacientes inscritos, lista de espera, atendimento, alta, encaminhamentos, abandono e desistência); e aspectos do tratamento (abordagens teóricas, tipos de atendimento e número de sessões realizadas, faltas). 
Em relação aos aspectos de procura de atendimento, foi utilizada uma adaptação do roteiro de Campezatto (2006) para a classificação dos motivos de procura e queixas trazidas pelos responsáveis, classificados da seguinte forma: 1) dificuldades em processos cognitivos; 2) dificuldades no comportamento afetivo; 3) dificuldades de relacionamento interpessoal; 4) dificuldades na vida diária; 5) dificuldades na esfera sexual; 6) sintomas físicos; 7) distúrbios orgânicos; 8) distúrbios de alimentação ou sono; 9) dependência química; 10) distúrbios psiquiátricos.

Após o levantamento de todos os prontuários e identificação de todas as queixas, foi feita uma segunda classificação dos motivos de procura de atendimento nas seguintes categorias: 1) Motivos relacionados à escola - categoria em que foram incluídos todos os motivos relacionados a problemas de aprendizagem, dificuldades cognitivas e sofrimentos no ambiente escolar; 2) Motivos relacionados a problemas afetivos e de relacionamento categoria em que foram incluídos os motivos classificados como transtornos de conduta e emocionais, e, também, de dificuldades de relacionamento interpessoal; 3) Problemas somáticos - categoria em que foram consideradas as variáveis associadas às manifestações de sintomas somáticos, distúrbios na alimentação, sono e fala; 4) Outros - dificuldades familiares; dificuldades relacionadas à sexualidade; distúrbios psiquiátricos.

Ainda em relação aos motivos de encaminhamento, foram classificados tanto o motivo principal como também o somatório de todas as queixas apresentadas sobre uma mesma criança, já que muitas crianças apresentaram queixas combinadas. Os responsáveis pela procura por atendimento psicológico infantil geralmente apresentam mais de uma queixa relativa ao comportamento da criança (Silvares, 1993; Santos \& Alonso, 2004). Finalmente, quanto à procedência dos encaminhamentos para atendimento psicológico, foram identificados os seguintes locais: 1) escolas; 2) serviços médicos e comunitários; 3 ) pediatra; 4) familiares; 5) judiciário.

Os dados foram analisados por meio de estatística descritiva, utilizando-se o programa SPSS for Windows, versão 15. Foram utilizadas análises descritivas e associativas mediante o teste Quiquadrado para medidas não paramétricas e o Teste ANOVA para variáveis contínuas.

\section{Procedimentos éticos}

A clínica-escola de psicologia pesquisada possui a prática de colher autorização para futuros estudos em um Termo de Consentimento Livre e Esclarecido de todos pacientes, já no ato da inscrição para atendimento psicológico. Por esse motivo, não houve necessidade de contato com os participantes para a realização desta pesquisa.

\section{Resultados}

Do total de 899 pacientes atendidos no período de 1999 a 2006, verificou-se que 499 (51,76\%) casos foram procura por atendimento psicológico por crianças. Destes, 212 (42,5\%) receberam e $287(57,5 \%)$ não receberam atendimento. Cento e cinco $(36,5 \%)$ desistiram quando chamadas e 182 $(63,5 \%)$ não tinham perfil (renda superior ao exigido, não foram localizadas, ou foram reencaminhadas). 0 tempo de permanência em lista de espera foi de 13 meses em média.

As características familiares em termos de renda indicaram que $30 \%$ das famílias situavam-se no grupo que tinha renda de até um salário-mínimo, $40 \%$ tinham renda de até dois salários e as restantes de até quatro salários-mínimos. Verificou-se que 53\% das crianças provinham de famílias intactas. As demais crianças, $32 \%$ residiam com as mães e o restante com os pais ou familiares.

A procura por atendimento em relação às faixas etárias indicou que a frequência entre 2 e 5 anos foi de 54 casos (10,8\%); entre 6 e 9 anos, de 281 casos $(56,4 \%)$; e entre 10 e 12 anos, 164 casos $(32,9 \%)$. Especificamente, as idades de maior procura foram a de 7 anos (18\%), seguida da idade de 8 anos $(15,2 \%)$. Em relação ao sexo das crianças, os meninos foram os que mais procuraram atendimentos (67\%) em comparação com a procura de meninas (32\%). Entretanto, considerando-se a distribuição por sexo e faixa etária, observa-se que no primeiro grupo (entre 2 e 5 anos) não há diferenças entre os meninos e as meninas em relação à procura por atendimento. Esta diferença aumenta nos dois outros grupos etários. Os meninos constituem 70,8\% da procura na faixa etária entre 6 e 9 anos e na faixa etária entre 10 e 12 anos $(65,9 \%)$.

Quanto à procedência dos encaminhamentos, $63,5 \%$ das crianças foram enviadas por escolas, 
seguido dos familiares $(10,4 \%)$, serviços médicos comunitários $(9,4 \%)$, pediatras $(9 \%)$ e departamento jurídico $(6,2 \%)$. Na faixa etária entre 2 e 5 anos, a maioria dos encaminhamentos foi feita por pediatras $(26,7 \%)$ e familiares $(25 \%)$; já a escola é responsável por $90 \%$ dos encaminhamentos nas idades entre 6 e 12 anos. Os principais motivos de procura foram problemas afetivos e de comportamento ( $\mathrm{n}=217,43,5 \%)$, dificuldades relacionadas à escola ( $\mathrm{n}=160,32,2 \%)$, outros $(\mathrm{n}=66,13,2 \%)$ (dificuldades familiares, dificuldades relacionadas à sexualidade, distúrbios psiquiátricos) e problemas somáticos e ansiedade ( $\mathrm{n}=56,11,2 \%)$.

Entre os casos atendidos, a média de atendimentos psicoterápicos realizados foi de 17 sessões (d.p. = 20). A mediana da distribuição da frequência de atendimentos foi $\mathrm{Md}=9$, indicando que $50 \%$ dos casos foram atendidos entre uma a nove sessões de psicoterapia. Dentre as 212 crianças atendidas, verificou-se que $136(64,15 \%)$ abandonaram o tratamento.

\section{Análise dos casos de abandono de atendimento psicológico}

Entre as 136 crianças que abandonaram seus atendimentos psicológicos, 81 (58,8\%) frequentaram 1 a 5 sessões; 27 (19,8\%), 6 a 15 sessões; 14 $(10,7 \%), 16$ a 25 sessões, $14(10,7 \%), 26$ sessões. A média de sessões entre as crianças que abandonaram seus atendimentos psicológicos foi $\mathrm{M}=9,80$ (d.p. $=12,87)$ e entre as que tiveram alta, 37,77 sessões (d.p. $=22,81)$. Um dado importante é que $41,7 \%(\mathrm{n}=55)$ dos abandonos ocorriam ainda no processo de avaliação dos pacientes, verificando-se que, após a décima sessão, a probabilidade para o abandono caía significantemente.

A análise por meio do teste Qui-quadrado das principais características sociodemográficas investigadas em relação à criança, sexo, idade e escolaridade; e dados da família, idade dos pais, escolaridade, renda familiar em relação ao abandono de terapia, apontou as seguintes características. Entre as crianças que abandonaram seus atendimentos psicológicos, $80(58,5 \%)$ eram meninos e 56 $(41,5 \%)$ meninas, não havendo diferença significativa entre os sexos (qui-quadrado $=7,43, p=0,11$ ) . A média de permanência em atendimento das meninas foi $M=9,13$ (d.p. $=12,80$ ) e dos meninos $M=10,29$ (d.p. $=12,97$ ). Tanto para meninos quanto para meninas, a maior ocorrência de abandonos ocorreu entre os 6 e 9 anos de idade $(n=74,54,4 \%)$, seguida da faixa etária entre 10 e 12 anos ( $\mathrm{n}=45$, $33,2 \%$ ), quando ocorre a transição da infância para puberdade. Em relação à escolaridade das crianças, verificou-se que $51 \%(\mathrm{n}=54)$ estavam cursando as séries iniciais do Ensino Fundamental, ou seja, período de inicialização escolar e social.

A análise do abandono de terapia em ralação à idade paterna, materna e do responsável em relação ao abandono inicial (AI), intermediário (AIT) e tardio (AT) indicou que crianças com pais jovens (entre 20 e 30 anos) abandonavam mais frequentemente o tratamento na fase inicial (qui-quadrado $=$ $9,66, d f=4, p=0,05)$. Não houve diferenças entre as idades dos responsáveis e das mães.

Em relação à escolaridade dos pais, mães e responsáveis, somente 91 pais informaram a escolaridade, sendo que destes, 49,5\% possuíam o Ensino Fundamental incompleto. Em relação às mães, $54,6 \%(\mathrm{n}=58)$ possuíam o Ensino Fundamental incompleto. Quanto aos responsáveis ( $\mathrm{n}=20), 60 \%$ tinham Ensino Fundamental incompleto. Não houve diferenças entre o nível de escolaridade paterna ou materna e a ocorrência de abandonos.

Referente à renda familiar das famílias dessas crianças, $44,7 \%(\mathrm{n}=42)$ possuíam renda igual ou inferior a um salário-mínimo, 43,6\% (n = 41) tinham renda de dois salários-mínimos, e 11,7\% ( $\mathrm{n}=11$ ), acima de três salários-mínimos. Quarenta e duas famílias não informaram a renda.

Em relação aos locais de encaminhamento, observou-se que 95 casos $(70,9 \%)$ corresponderam a abandonos de crianças encaminhadas pela escola, seguidos por encaminhamentos vindos de serviços médicos e comunitários ( $\mathrm{n}=13,9,7 \%$ ), departamentos jurídi$\cos (n=12,9 \%)$, familiares $(n=9,1,3 \%)$ e pediatras ( $\mathrm{n}=5,0,7 \%$ ). Comparando-se o abandono de terapia em relação ao encaminhamento de toda a clientela infantil inscrita, verificou-se que o maior índice de abandono ocorreu entre crianças provenientes da escola e crianças dos departamentos jurídicos (qui-quadrado = $26,91, d f=16, p=0,04)$. Ao contrário, o menor número de abandonos ocorreu entre as crianças provenientes de encaminhamento familiar.

As crianças encaminhadas para atendimento apresentavam uma média de $M=2,77$ (d.p. = 1,66) aos motivos de encaminhamento. Não houve diferenças entre os sexos ou categorias de idade em relação aos motivos de atendimento e abandono de 
terapia. Considerando-se o somatório de todos os motivos mencionados, verificou-se que os problemas afetivos e de relacionamento (transtornos de conduta e emocionais, dificuldades de relacionamento interpessoal) foram mencionados em 47,2\% das crianças. Os motivos relacionados à escola (motivos relacionados a problemas de aprendizagem, dificuldades cognitivas e sofrimentos no ambiente escolar) foram mencionados por $24,6 \%$ das crianças. Entre as crianças com motivos relacionados à escola, $46,4 \%$ referiam-se a queixas quanto ao desinteresse escolar ou vivências de sofrimento em permanecer no ambiente escolar, e 53,6\% referiam-se a dificuldades cognitivas ou de aprendizagem. Os problemas somáticos (sintomas somáticos, distúrbios na alimentação, sono e fala) foram citados por $10,7 \%$ das crianças. Finalmente, dificuldades familiares, dificuldades relacionadas à sexualidade e distúrbios psiquiátricos foram mencionadas por $17,5 \%$ das crianças. A maior frequência de abandonos ocorreu entre as crianças encaminhadas por motivos escolares (qui-quadrado $=59,24, \mathrm{df}=12$, $p=0,00)$. Não foram identificadas diferenças entre o período de abandono (inicial, intermediário e tardio) e os motivos de encaminhamento.

Em relação aos familiares do paciente, 90 $(77,5 \%)$ crianças foram acompanhadas pelas mães nas consultas e $12(10,4 \%)$ por ambos os pais. Das demais crianças, $34,4 \%(n=44)$ moravam somente com as mães, 3,9\% (5) moravam somente com o pai, 3,9\% $(n=5)$ moravam com parentes maternos, e 4,7\% $(n=6)$ moravam com responsáveis adotivos. Utilizando-se o teste Qui-quadrado, constatou-se que, em relação à moradia, não houve diferenças na frequência de abandono inicial (AI) quanto a morar com os pais, somente com o pai, com parentes e com famílias adotivas. Entretanto, houve maior ocorrência de abandono inicial (AI) no grupo de crianças que morava somente com as mães (qui-quadrado = $15,74, \mathrm{df}=8, \mathrm{p}=0,04)$.

Entre as crianças que abandonaram seus atendimentos psicológicos, verificou-se que, $58,2 \%$ $(\mathrm{n}=80)$ foram chamadas de listas de espera e $41,8 \%(n=56)$ foram atendidas no momento da inscrição. Entre as crianças que ficaram em lista de espera, $71,8 \%(n=57)$ abandonaram no início do atendimento psicológico (até nove sessões). Comparando-se o tempo de espera, as crianças que permaneceram até nove sessões $(n=69)$ ficaram em espera em média oito meses, as que ficaram de
10 a 25 sessões $(n=20)$ tiveram uma média de cinco meses de espera.

Quanto às faltas dos pacientes nas consultas agendadas durante $o$ atendimento psicológico, observou-se que $82 \%(n=111)$ das crianças tiveram entre uma e três faltas pelo menos, $13,3 \%(n=17)$ tiveram de 4 a 7 faltas, e 4,8\% (n $=8)$ tiveram mais do que sete faltas. Um índice de $53,2 \%(n=72)$ dos pacientes apresentava em média três faltas em um período próximo ao abandono. Também se observou que os abandonos não ocorreram em períodos de retorno de recesso da clínica-escola (julho, janeiro e fevereiro), mas em outros meses do ano $(88,7 \%$, $\mathrm{n}=120$ ). Em relação ao cuidado dos profissionais (monitores, professores e estagiários) com os registros, foi encontrado, entre todo o material manuseado, um total de 83,4\% ( $\mathrm{n}=113$ ) dos prontuários em um estado de organização adequado quanto a dados e informações gerais do atendimento.

Constatou-se que 97,7\% ( $\mathrm{n}=130)$ dos atendimentos não foram submetidos à troca de estagiário/psicólogo. Os atendimentos foram realizados, em sua maioria, por estagiários 78,3\% ( $\mathrm{n}=106)$ e os demais por profissionais, supervisores, sendo $91,9 \%(n=125)$ atendimentos individuais. Entre as abordagens teóricas do local, os atendimentos foram realizados em sua maioria pela Psicoterapia de Orientação Psicanalítica ( $\mathrm{n}=85,62,9 \%), 27$ casos $(19,8 \%)$ foram atendidos pela Terapia Cognitivo-comportamental e $23(17,2 \%)$ pela Terapia Fenomenológica Existencial. Não houve diferenças de abandono em relação às abordagens teóricas.

\section{Discussão}

Vários estudos ressaltam os altos índices de abandonos em psicoterapia infantil, mas as razões para isso são raramente procuradas entre as famílias e os psicólogos (Stevens, Kelleher, Ward-Estes \& Hayes, 2006). Os resultados desta pesquisa identificaram que a faixa etária infantil, além de constituir o grupo de maior procura para atendimentos psicológicos em clínicas-escola, possui também os maiores índices de abandono psicoterápico. No presente estudo este índice foi de $64,2 \%$. Portanto, esses resultados são superiores aos de Romaro e Capitão (2003), que encontraram um índice de $45,5 \%$ de abandonos por crianças numa clínica-escola da região de São Paulo (SP), bem como ao de Lhullier 
(2002), que encontrou um índice de 51,8\% na região de Pelotas (RS).

A questão do abandono também é mencionada na literatura internacional, indicando que este problema não se restringe ao contexto nacional. Luk et al. (2001), por exemplo, numa investigação em clínicas urbanas e suburbanas nos EUA, também mencionam o alto índice de abandono, entre 30 a 40\%, nos atendimentos em clínicas de crianças e adolescentes.

No geral, os estudos não identificam uma variável isolada como responsável para o abandono de crianças em psicoterapia (Luk et al., 2001; Stevens, Kelleher, Ward-Estes \& Hayes, 2006). Considerando as características sociodemográficas, alguns aspectos notados nas características do grupo de crianças que abandonam o atendimento foram semelhantes a outros estudos nacionais. Destaca-se a grande procura de atendimento por crianças do sexo masculino e na faixa etária escolar (Melo \& Perfeito, 2006; Romaro \& Capitão, 2003; Santos \& Alonso, 2004; Silvares, 2006a). Entretanto, em relação ao abandono, embora os meninos também tenham apresentado neste estudo predominância em relação à procura por atendimentos psicológicos, não houve diferenças quanto ao sexo da criança em relação ao abandono. Além disso, um pouco mais da metade das crianças possuía entre 6 e 9 anos de idade e cursava um dos dois primeiros anos do Ensino Fundamental, ou seja, uma fatia da amostra significante, pertencente à inicialização escolar e socialização na infância. De tal maneira, essa faixa etária além de corresponder à maior procura por atendimentos psicológicos nos primeiros anos de escolarização, é também a que mais abandona o atendimento psicológico (Romaro \& Capitão, 2003; Shoen-Ferreira, Silva, Farias \& Mattos, 2002).

Assim, esses períodos de transições na infância, como o início da socialização escolar ou da puberdade, caracterizam-se por momentos de maior de vulnerabilidade pelas demandas psicológicas correspondentes a maior independização. Portanto, constituem-se como períodos evolutivos que devem ser considerados pelos terapeutas como fatores de risco para o abandono, sendo importante realizar avaliações cuidadosas da evolução da aliança terapêutica nestas faixas etárias.

Considerando-se o aspecto das características familiares, observou-se que houve maior frequência de abandono entre aquelas que residiam somente com a mãe. Outros estudos, como Kazdin (1990) igualmente identificaram, numa investigação sobre o abandono de tratamento por crianças, uma maior prevalência de abandonos por filhos de mães solteiras. Famílias monoparentais enfrentam situações importantes de maior estresse no cuidado dos filhos. Entretanto, não é a situação familiar em si que está associada à maior vulnerabilidade, mas, principalmente questões de renda financeira e trabalho (Gallo \& Williams, 2008). Outro aspecto interessante refere-se à idade dos pais. Mais da metade dos pais das crianças possuía entre 20 e 35 anos de idade. Luk et al. (2001), também em um estudo sobre abandono de tratamento infantil, encontraram altos índices de abandonos associados aos pais jovens. Pode-se supor que o alto índice de procura e de abandonos psicoterápicos por crianças de pais e mães jovens associe-se ao despreparo parental ou imaturidade no processo de criação dos filhos (Cicco, Paiva \& Gomes, 2005).

Por último, questões do nível educacional e renda das famílias também foram aspectos importantes de caracterização do grupo investigado. 0 baixo nível de escolaridade e renda dos pais são situações frequentemente identificadas em alguns estudos sobre clientela de clínica-escola (Silvares, Meyer, Santos \& Gerencer, 2006b). Santos e Alonso (2004), em um estudo sobre caracterização da clientela numa clínica-escola de psicologia, encontraram que a maioria dos pais possuía até oito anos de estudo. Da mesma forma, Shoen-Ferreira et al. (2002) mencionam que o perfil e as queixas de clientes em clínica-escola indicam que os pais da maioria das crianças encaminhadas possuem baixo nível de escolaridade. Além da baixa escolaridade presente nesses familiares, a maioria possui uma renda mensal de até dois salários-mínimos. Também Santos e Alonso (2004), ao realizarem uma caracterização da demanda infantil para atendimento psicológico, encontraram que a maioria das famílias possuía renda entre um e três salários-mínimos, com baixo nível socioeconômico e social (Romaro \& Capitão, 2003; Santos \& Alonso, 2004).

A escola foi o local que mais referiu crianças para atendimento psicológico. No geral, a literatura aponta que a maioria das crianças é encaminhada com queixas de insucesso escolar (Romaro \& Capitão, 2003), sendo a escola e a família os locais que mais encaminham crianças para tratamento (Santos \& Alonso, 2004). No presente trabalho, a família não 
foi um local predominante em relação à procedência das crianças, mas observou-se que as crianças encaminhadas pelas famílias abandonaram menos o tratamento. Já entre as crianças encaminhadas por escolas houve maior abandono de atendimento, o que também foi observado nas crianças provenientes de encaminhamentos do judiciário. Nessas situações, frequentemente, a demanda de atendimento é percebida pela família como imposta, não havendo uma apropriação e identificação conjunta do motivo do atendimento. Ao contrário, quando as famílias tomam iniciativa e procuram atendimento para suas crianças, o estabelecimento da aliança terapêutica é mais efetivo (Silvares, 1993).

Entre os motivos de procura para atendimento, a maioria das situações referia-se a queixas relacionadas a problemas afetivos e de relacionamento, associados a dificuldades sobre a conduta, problemas emocionais e dificuldades de relacionamento interpessoal. A segunda queixa predominante, presente em mais da metade da amostra, foi relacionada a problemas escolares. Entretanto, uma análise mais detalhada dos prontuários indicou que somente a metade dessas queixas refletia desinteresse da criança pela escola ou por sofrimento no ambiente escolar (dificuldade de separar-se da mãe ou família, medo, resistência ao ambiente escolar, timidez, choro na escola, isolamento na escola), estando a outra parte relacionada a problemas de aprendizagem ou dificuldades cognitivas. Alguns estudos relatam que há poucas crianças que realmente possuem problemas cognitivos, embora as escolas encaminhem com o rótulo de problemas de aprendizagem ou fracasso escolar (Shoen-Ferreira et al. 2002; Santos \& Alonso, 2004; Melo \& Perfeito, 2006). Finalmente, a terceira queixa predominante referia-se a dificuldades familiares (violência familiar, doenças, separação conjugal etc.).

Entre as crianças que ficaram em lista de espera, grande parte abandona o atendimento no início (até 9 sessões). Hynan (1990) salienta que pacientes em lista de espera diminuem a motivação inicial pela demora da consulta. Alguns estudos indicam que a lista de espera em locais de assistência a populações mais carentes deve ser menor possível para diminuir a possibilidade de abandono (Bueno et al., 2001). Além do abandono inicial, as listas de espera prejudicam outros aspectos, tal como no caso do número expressivo de crianças que desistem da procura por atendimento quando chamadas.
Romaro e Capitão (2003) encontraram também um número significante de crianças que, depois de inscritas, desistiram do interesse pelo tratamento quando chamadas. Por esses motivos, é importante que as clínicas-escola tenham as listas sob controle quanto ao tempo de espera para reduzir os riscos relacionados à demora pelos serviços psicológicos necessitados.

Quanto às variáveis da instituição e do tratamento, foi frequente a ocorrência de crianças que abandonaram o atendimento ainda na avaliação inicial. Em termos clínicos, esses achados apontam a necessidade de as clínicas-escola atentarem para o processo de recepção de um paciente, pois o momento inicial parece ser uma das situações mais delicadas do atendimento (Campezatto \& Nunes, 2007). Para Perfeito e Melo (2004), as consultas iniciais em atendimento psicológico infantil precisam ser utilizadas para conscientizar os pais sobre as dificuldades presentes, para que aumente as chances de permanecerem no atendimento, facilitando o engajamento ao tratamento e tornando-os responsáveis pelos seus problemas.

Vários estudos apontam o alto índice de abandono por crianças até a décima sessão (Lhullier, 2002; Campezatto \& Nunes, 2007; Prebianchi \& Cury, 2005; Vargas, 2002). Lhullier (2002) relata que a maioria dos pacientes que abandonam o atendimento comparece somente na primeira sessão ou até a oitava sessão. Vargas (2002) aponta um número ainda menor de sessões, indicando que grande parte dos pacientes abandona seu atendimento psicológico até a terceira sessão.

No presente estudo, grande parte das crianças compareceu em uma a quatro sessões, abandonando o processo já na etapa inicial do atendimento, quando nem mesmo o próprio processo de avaliação foi concluído. Assim, verifica-se que a maioria das famílias abandona antes mesmo de tomar conhecimento do diagnóstico e do tratamento adequado ao problema do filho. As crianças pela sua posição de dependência ficam desassistidas e vulneráveis a novas patologias físicas ou mentais, pelo sofrimento existente. Para evitar o abandono já na etapa de avaliação, Bueno et al. (2001) ressaltam a importância do acolhimento inicial em atendimentos psicológicos, efetuando um adequado esclarecimento para a família sobre questões do processo terapêutico, bem como falando sobre as expectativas, as questões do contrato, as explicações básicas 
sobre o percurso de um tratamento psicológico. Enfim, possuindo uma intervenção mais organizada e acolhedora, principalmente no início dos atendimentos, quando os abandonos mais ocorrem.

Entre as abordagens teóricas do local, ainda verifica-se uma preferência pelos profissionais e pelos estudantes de psicologia em estágio clínico pela abordagem teórica da Psicoterapia de Orientação Psicanalítica. Entretanto, a técnica é menos importante, pois é o contexto interpessoal que irá contribuir para a aderência ao tratamento e também à capacidade para formar vínculos, para uma futura aliança terapêutica adequada ao processo psicoterápico (Lhullier, 2002; Lhullier et al., 2006). Se as famílias experimentarem uma aliança positiva, sentindo segurança e compreensão pelo profissional que atende ao filho, o índice de abandono em psicoterapia infantil é menor.

No caso de psicoterapia infantil, não depende da criança a iniciativa em comparecer ou ir até o local de atendimento, ficando evidente a importância do estabelecimento da aliança terapêutica com a família. Dessa maneira, os profissionais devem estar atentos a questões intra e interpsíquicas no atendimento infantil, correlacionando as posições parentais e a sintomatologia infantil, as condições mentais maternas e paternas, a estrutura e o funcionamento das famílias, as expectativas dos pais em relação ao filho e a percepção da necessidade de ajuda psicológica (Melo \& Perfeito, 2006). Logo, o terapeuta deve escutar a ambos os pais para investigar e compreender a visão de cada um e em que nível esta poderá facilitar ou dificultar o processo terapêutico, direcionando um olhar para a relação do casal quanto à educação do filho, às próprias questões do casal e aos papéis que cada um ocupa no grupo familiar (Glenn Sabot \& Bernstein, 1996; Lisondo, Scazufka, Ribeiro, Noto, Inglez de Souza \& Franch, 1996; Cunha \& Benetti, 2007). Cada participante do processo terapêutico deve receber intervenções específicas para o sucesso do atendimento psicológico, enfatizando as expectativas e o comparecimento no atendimento psicológico (Shuman \& Shapiro, 2002).

Além dos aspectos familiares, também devem ser abordadas as questões institucionais como as habilidades interpessoais e a capacidade em formar vínculos nos técnicos e estagiários de psicologia (Lhullier, 2002; Lhullier et al., 2006). Os profissionais devem possuir, principalmente em clínicas-escola, local de prática, atendimento, ensino e pesquisa, aspectos como flexibilidade, criatividade e interesse na otimização dos procedimentos do atendimento em saúde pública psicológica (Perfeito \& Melo, 2004). Sendo assim, o desenvolvimento de intervenções grupais pelas instituições de atendimento, respeitando critérios terapêuticos pode ser uma alternativa importante e com resolutividade mais eficiente, levando em conta o tempo de permanência em lista de espera associado à desistência do atendimento (Santos \& Alonso, 2004; Silvares, 2000).

Em suma, são diversas as variáveis associadas ao abandono psicoterápico nessa faixa etária. Esses diferentes elementos são aspectos que devem ser incluídos no desenvolvimento de pesquisas, visando melhorar o conhecimento e evolução da psicoterapia infantil. Segundo Bueno et al. (2001), identificar preditores para abandono abre caminhos para ações preventivas, pois os psicólogos clínicos devem concentrar-se em novos formatos e novos conhecimentos sobre medidas que atuem no nível clínico e assistenciais. Para tal, a implantação de estudos voltados para a avaliação do processo de atendimento, desde as sessões iniciais de acolhimento até o encaminhamento final, é uma ação vital para o conhecimento dos atendimentos prestados e fornecimento de informações que subsidiem as intervenções e o melhor preparo dos alunos em seu treinamento.

\section{Referências}

Bueno, H. A., Córdoba, D. J., Escolar, P. A., Carmona, C. J., \& Rodríguez, G. C. (2001). El abandono terapéutico. Actas Espain Psiquiatría, 29(1), 33-40.

Campezatto, P. V. M. (2006). As Clínicas-Escola de cursos de Psicologia da Região Metropolitana de Porto Alegre. Dissertação de Mestrado. Instituto de Psicologia. Universidade Católica do Rio Grande do Sul, Porto Alegre, Brasil.

Campezatto, P. V. M., \& Nunes, M. L. T. (2007). Atendimento em clínicas-escola de psicologia da região metropolitana de Porto Alegre. Estudos de Psicologia, 24(3), 363-374.

Chilelli, K. B., \& Enéas, M. L. E. (2000). Desistência em psicoterapia breve: Pesquisa documental e da opinião do Paciente. Boletim de Iniciação Científica de Psicologia, 1(1), 47-52. 
Cicco, M. F., Paiva, M. L. S. C., \& Gomes, I. C. (2005). Família e conjugalidade: 0 sintoma dos filhos à imaturidade do casal parental. Psicologia Clínica, 17(1), 53-63.

Cunha, T. R. S, \& Benetti, S. P. C. (2007). Casais com filhos e a qualidade da satisfação conjugal. XXXVII Reunião Anual de Psicologia - Sociedade Brasileira de Psicologia/Florianópolis, São Paulo.

Freitas, F. A., \& Noronha, A. P. P. (2005). Clínica-Escola: Levantamento de instrumentos utilizados no processo psicodiagnóstico. Psicologia Escola e Educação, 9(1), 87-93.

Gallo, A. E., \& Williams, L. C. A. (2008). A escola como fator de proteção à conduta infracional de adolescentes. Cadernos de Pesquisa, 38(133), 41-59.

Glenn, J., Sabot, L. M., \& Bernstein, I. (1996). Psicanálise e psicoterapia de crianças. In J. Glenn (Org.). O papel dos pais na análise de crianças. (pp. 239-257). Porto Alegre: Artes Médicas.

Godman, S. W. (2003). A comparison of completers and noncompleters of exposure-based cognitive and behavioral treatment for phobic and anxiety disorders in youth. Journal Psychology, 71(1), 701-705.

Green, J. (2005). Annotation: The therapeutic alliance A significant but neglected variable in child mental health treatment studies. Journal of Child Psychology and Psychiatry, 47(5), 425-435.

Hynan, D. J. (1990). Clients reasons and experiences in treatment that influence termination of psychoterapy. Universidade do Rio Grande do Norte. Journal Clinical Psychology, 46(1), 891-895.

Kelleher, K., Ward-Estes, J., \& Hayes, J. (2006). Perceived barriers to treatment and psychotherapy attendance in child community mental health centers. Community Mental Health Journal, 42(5), 449-458.

Kazdin, A. E. (1990). Premature termination from treatment among children referred for antisocial behavior. Journal Clinical Psychology, 31(3), 415-425.

Lisondo, A. B. D., Scazufka, E., Ribeiro, M., Noto, I. S. B. S., Inglez De Souza, M. S., \& Franch, N. J. P. (1996). Psicanálise de crianças: um terreno minado? Revista Brasileira de Psicanálise, 30(1), 9-26.

Löhr, S. S., \& Silvares, E. F. M. (2006). Clínica-escola: Integração da formação acadêmica com as necessidades da comunidade. In E. F. M. Silvares (Org.). Atendimento psicológico em clínicas-escola. (pp. 1222). Campinas: Alínea.
Lhullier, A. C. (2002). Abandono de tratamento em psicoterapias realizadas numa clínica-escola. Tese de Doutorado publicada. Faculdade de Psicologia, Pontifícia Universidade Católica do Rio Grande do Sul, Porto Alegre, Brasil.

Lhullier, A. C., Nunes, M. L. T., \& Lessa, H. (2006). Preditores de abandono de psicoterapia em pacientes de clínica-escola. In E. F. M. Silvares. (Org.). Atendimento Psicológico em Clínicas-escola. (pp. 227-246). Campinas: Alínea.

Louzada, R. C. R. (2003). Caracterização da clientela atendida no núcleo de Psicologia aplicada da Universidade Federal do Espírito Santo. Estudos de Psicologia, 8(3), 451-457.

Luk, E. S. L., Staiger, P. K., Mathai, J., Wong, L., Birleson, P., \& Adler, R. (2001). Children with persistent conduct problems who dropout of treatment. European Child and Adolescent Psychiatry, 10(1), 28-36.

Melo, A. P. S., \& Guimarães, M. D. C. (2005). Factors associated with psychiatric treatment dropout in a mental health reference center. Revista Brasileira Psiquiatria, 27(2), 113-118.

Melo, S. A., \& Perfeito, H. C. C. S. (2006). Características da população infantil atendida em triagem no período de 2000 a 2002 numa clínica-escola. Estudos de Psicologia, 23(3), 239-249.

Perfeito, H. C. C. S., \& Melo, S. A. (2004). Evolução dos processos de triagem psicológica em uma clínica-escola. Estudos de Psicologia, 21(1) 33-42.

Piper, W. E., Ogrodniczuk, J. S., Joyce, A. S., McCallum, M., Rosie, J. S., O'Kelly, J. G., et al. (1999). Prediction of dropping out in time-limited, interpretive individual. Psychotherapy, 36, 114-122.

Prebianchi, H. B., \& Cury, V. E. (2005). Atendimento infantil numa clínica-escola de Psicologia: Percepção dos profissionais envolvidos. Paidéia, 15(31), 249-258

Romaro, R. A., \& Capitão, C. G. (2003). Caracterização da clientela da clínica-escola de Psicologia da Universidade de São Francisco. Psicologia Teoria $e$ Prática, 5(1), 111-121.

Russel, R. L., \& Orlinsky, D. E. (1996). Psychotherapy research in historical perspective. Archives of Generl Psychiatry, 53(8), 708-715.

Santos, W. P., \& Alonso, M. Z. (2004). Caracterização da demanda infantil de um serviço de Psicologia. Revista do Ministério da Saúde Pública, 3(1), 35-42. 
Shoen-Ferreira, T. H., Silva, D. A., Farias, M. A., \& Mattos E. F. (2002). Perfil e principais queixas dos clientes encaminhados ao centro de atendimento e apoio Psicológico ao adolescente. Psicologia em Estudo, 7(1), 73-82.

Shuman A L., \& Shapiro, J. P. (2002). The effects of preparing parents for child psychotherapy on accuracy of expectations and treatment. Community Mental Health Journal, 38(1), 3-16.

Silvares, E. F. M. (1993). O papel preventivo das clinicas-escola de psicologia em seu atendimento a Crianças. Temas em Psicologia, 2(1), 87-97.

Silvares, E. F. M. (2000). Invertendo o caminho tradicional numa clínica-escola de Psicologia. Estudos de Psicologia, 5(1), 149-180.
Silvares, E. F. M. (2006a). Atendimento Psicológico em Clínicas-escola. Campinas: Alínea.

Silvares, E. F. M., Meyer, S. B., Santos, E. O. L., \& Gerencer, T. T. (2006b). Um estudo em cinco clínicas-escola brasileiras com a lista de verificação comportamental para crianças- CBCL. In E. F. M. Silvares (Org.). Atendimento Psicológico em Clínicas-escola. Campinas: Alínea.

Stevens, J., Kelleher, K., Ward-Estes, J., \& Hayes, J. (2006). Perceived barriers to treatment and psychotherapy attendance in child community mental health centers. Community Mental Health Journal, 42(5), 449-458.

Vargas, F. (2002). Abandono de psicoterapia em instituição de formação de psicoterapeutas. Dissertação de Mestrado. Faculdade de Psicologia, Pontifícia Universidade Católica do Rio Grande do Sul, Porto Alegre. 